\title{
La participación social de la comunidad educativa en escenarios complejos de educación media en un liceo de Venezuela
}

\author{
The social participation of the educational community in complex \\ settings of secondary education in a high school in Venezuela
}

\section{Félix Heredia Rojas 1}

\section{Revista}

\section{Educación y Sociedad}

Citar como: Heredia, F. (2020). La participación social de la comunidad educativa en escenarios complejos de educación media en un liceo de Venezuela. Revista Educación y Sociedad, 01(02), 55-70. doi: 10.53940/reys.v112.59

Artículo recibido: 29-06-2020 Artículo aprobado: 20-11-2020 Arbitrado por pares

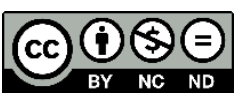

\section{ACEES}

\section{Resumen}

El presente trabajo, tuvo por objetivo generar una aproximación teórica acerca de la participación social de una comunidad educativa en escenarios complejos de educación media en Venezuela. Se orientó en el paradigma interpretativo con un enfoque metodológico cualitativo, perspectiva epistémica fenomenológica y método fenomenológico hermenéutico; con tres informantes clave de una institución. Como técnica de recolección de información se utilizó la entrevista semi estructurada, la cual se codificó, categorizó, estructuró e integró. Luego se contrastó y trianguló, lo cual permitió proponer la incorporación a la acción escolar, al modelo educativo vigente, un quinto pilar, referido a aprender a participar, como médula espinal de esta aproximación teórica.

\section{Palabras clave: participación social, escenarios educativos complejos}

\section{Abstract}

The objective of this work was to generate a theoretical approach to the social participation of the educational community in complex middle education scenarios in Venezuela. It was oriented in the interpretive paradigm with a qualitative methodological approach, phenomenological epistemic approach and hermeneutical phenomenological method; with three key informants from an institution. The semi-structured interview was used as the information gathering technique, which was coded, categorized, structured and integrated; Then it was contrasted and triangulated, of which allowed us to propose the incorporation of a fifth pillar, referring to Learning to Participate, as the backbone of this Theoretical Approach, to the current educational model.

Key words: social participation, complex educational scenarios 


\section{Introducción}

La participación y el compromiso de la comunidad en la gestión educativa se considera como la interacción directa, personal y asociada a múltiples agentes, entre ellos, la familia, escuela, asociación de vecinos, organismos de desarrollo regional y nacional, entre otros, que permiten poner en práctica diferentes proyectos, programas y planes sustentados en estrategias integracionistas con participación ciudadana, comunitaria y social, protagónica, corresponsable y vinculada a la gestión del Estado, e impulsada por el Ministerio del Poder Popular para la Educación que aseguren y privilegien el desarrollo humano, familiar y comunitario establecido en la normativa legal del país.

Es por ello, que la realidad actual exige cambios profundos en la forma de concebir la educación en los nuevos escenarios complejos, derivados del proceso de globalización, presente hoy día en las distintas sociedades del mundo. De allí, surge la necesidad de educar al ciudadano para la participación social en esos nuevos contextos, que permita un proceso continuo y dinámico que implica una acción concientizadora y socializante; que produzca una movilización de la conciencia respecto a la circunstancia de vida, sus causas y efectos; que transmita patrones de comportamiento y nuevas formas de aprehender los detalles que ayuden optimizar la calidad del ambiente natural y humano.

En el caso específico de Venezuela, su Constitución (1999) plantea que la democracia es el eje rector que se expande a través del papel que la educación puede cumplir en un momento dado en la formación de y para la democracia como expresión de vida, además de su participación y protagonismo social. No obstante, se puede observar que la praxis educativa en las instituciones escolares apunta, al pensamiento individual y no colectivo, develando probablemente su exigua participación en la solución de problemas comunitarios y en el entorpecimiento de la construcción unificadora e integradora del conocimiento en sus miembros, acentuándose cada día más por las adversidades políticas, sociales y económicas que les aqueja.

Circunstancias que develan la necesidad de replantear la política educativa sobre la base de los documentos y diagnósticos que se han elaborado en los diferentes períodos de la educación, con el fin de propugnar la estimulación de una cultura de participación protagónica y corresponsable por parte de padres y/o representantes, educadores, educandos y demás organizaciones gubernamentales o no, que se relacionen directa o indirectamente con la escuela, para debatir y actuar sobre el ejercicio de la ciudadanía en el contexto educativo. Sobre esa base, surgió esta investigación acerca de la participación social de la comunidad educativa en escenarios complejos de educación media en Venezuela; siendo sus principales fundamentos teóricos el pensamiento complejo de Morín (1995) y la teoría crítica de la educación de Habermas (2000), como posturas que fundamentan y explican la temática abordada, a fin de comprender sus implicaciones.

Es necesario iniciar el discurso con el entendimiento de que es, en el campo de la educación donde son posibles interrelaciones coherentes y de sentido entre los individuos y el contexto donde se desenvuelven. Es así como, se puede decir que el fenómeno político más importante dentro de los actuales procesos de globalización que configuran al sistema - mundo, es la expansión y consolidación de la democracia, como marco de responsabilidad, compromiso e intervención significativa en las decisiones que afectan el orden social del planeta.

Esa realidad, plantea una situación de incertidumbre en virtud de los efectos generados por el proceso de acomodo y reacomodo de las economías mundiales; por el deseo de integración política, económica y social, descentralización y fragmentación. Este proceso desigual dirigido desde las grandes economías se revela como muy inequitativo, pensado desde otras fronteras y favoreciendo la aparición de bloques de poder que obstaculizan el desarrollo, sobre todo, en los países latinoamericanos como Venezuela. 
Díaz, Santisteban y Cascarejo (2013) señalan que la conformación de la aldea global no se explica únicamente por la emergencia de la fluidez comunicativa que ha propiciado el desarrollo de los mass media, también comporta la apertura de espacios de intervención efectiva, aunque todavía bastante limitada, en los asuntos locales e internacionales de las sociedades contemporáneas. En este orden de ideas, se puede señalar que uno de los mecanismos más importantes de la democracia, es la participación de los individuos en las decisiones, que tienen que ver con el aspecto político, económico y social de un país.

En tal sentido, el tema de la participación ha sido uno de los pilares fundamentales en las bases contextuales de las organizaciones de desarrollo social. Su comprensión, análisis y maneras de aplicación han constituido parte esencial de la reflexión y las funciones de estas organizaciones; lo que ha cobrado mayor vigencia, en tanto se observa como el término ha sido incorporado desde diversas perspectivas y enfoques de una manera explícita, en el quehacer de muchas otras instituciones y organizaciones tanto públicas como privadas. Tema que ha ido impregnando a sectores tan diversos como son el empresarial, el mundo de la corporación internacional o el académico, convirtiéndose en requisito o precepto para muchas de las actividades desarrolladas desde esos escenarios.

Al respecto, Santizo (2012) afirma que el impulso de la participación social en los centros educativos puede contribuir a obtener una educación de calidad, equidad, además de mejorar los resultados de aprendizaje en los estudiantes. Aunado a ello, puede favorecer la autonomía de esas instituciones y favorecer la democratización del sistema, elevando su calidad, tal como sostiene Zurita (2008) y Bloom (2010), quienes coinciden que puede lograrse con la ayuda de la participación social.

De allí, que en el contexto educativo se observa el establecimiento de una forma de relaciones sociales en la que los intereses se penetran recíprocamente, donde el progreso o reajuste merece una importante consideración en la conformación hacia una sociedad democrática más interesada que otras en organizar una educación que responda a sus intereses. Por tal razón, el fenómeno de la participación social es relevante en la medida que responda a los fines de quien la conduce y es irrelevante cuando en los procesos sólo se justifica. Por tal motivo, la escuela debe ser un lugar de tolerancia, de tareas asumidas y realizadas en grupo, de ejercicio concreto del respeto a los demás y de solución racional a las dificultades que toda convivencia conlleva.

De las apreciaciones antes expuestas, se puede presumir que existe el ánimo a la participación, pero la direccionalidad con la cual las autoridades de educación manejan el asunto, no es la más acorde con la realidad concreta de cada centro escolar, corriéndose el riesgo de perder la oportunidad de que sean los mismos actores del proceso que busquen soluciones a la problemática que vive la educación, tal como se vive en la educación media. Nivel de estudio en el que es difícil la integración de padres y representantes a las actividades escolares. Éstos únicamente asisten a retirar el resultado de evaluación de sus representados sin involucrarse en la resolución de ningún tipo de problema que aqueje la institución.

De allí, surge la inquietud de la realización de este trabajo sobre la participación social de la comunidad educativa en escenarios complejos de educación media en Venezuela por la inquietud de las siguientes interrogantes: ¿Qué significado le atribuyen los integrantes de la comunidad educativa a la participación social en escenarios educativos complejos de la educación media? ¿Cuáles son los constructos relacionados con la participación social de la comunidad educativa en escenarios complejos de educación media en Venezuela? Para dar respuesta, se planteó el siguiente objetivo general: a) generar una aproximación teórica acerca de la participación social de la comunidad educativa en escenarios complejos de educación media en un liceo del municipio Crespo (Estado Lara, Venezuela). Asimismo, se establecieron los siguientes objetivos específicos: a) interpretar el significado que le atribuyen los integrantes de la comunidad educativa a la participación social en escenarios educativos complejos de la educación media y b) develar los constructos teóricos relacionados con la participación social de la comunidad educativa en escenarios complejos de educación media. 
Este estudio, vislumbra la idea de retomar el proceso de participación social, como única vía válida de integración de todos los actores involucrados en dicho proceso. Por tal motivo, su relevancia se expresa a nivel teórico, por ofrecer un conocimiento a través de la concreción de conceptualizaciones o principios por medio de las propias interpretaciones que de él se desprendieron, al dar explicación de la participación social con el propósito de intentar llenar el vacío que existe en el contexto educativo.

Además, busca ofrecer soportes teórico metodológicos, como punto de partida, para construir nuevas propuestas que permitan vincular la participación con los contextos sociales educativos que demanden las propias necesidades, a través de elementos que pudieran coadyuvar a la reelaboración de otras teorías y modelos que favorezcan la participación social. De todo lo antes planteado, se desprenden las razones que justifican esta investigación, cuyo fin es generar una aproximación teórica ajustada a los cambios paradigmáticos que supone el tránsito por escenarios educativos complejos.

\section{Estudios previos relacionados con la investigación}

Producto de la revisión de trabajos previos relacionados con esta investigación, se seleccionó el realizado por Perales y Escobedo (2016) quienes realizaron una investigación en México con la finalidad de analizar la participación social en la educación: entre propuestas innovadoras y tradición educativa en aras de conocer las diversas normativas que buscan promover la capacidad organizativa y corresponsabilidad de padres de familia, estudiantes y agentes sociales. Ante lo cual, propusieron acciones que involucran a la comunidad y consejos escolares de participación social para propiciar un diálogo constructivo y corresponsable de la gestión y organización de la escuela.

Para ello, los autores antes mencionados se apoyaron en el paradigma interpretativo bajo el enfoque metodológico cualitativo y método etnográfico para indagar sobre la manera en que padres de familia, directivos y docentes se involucran en la constitución y operación de los consejos escolares en una escuela primaria. Los investigadores, coinciden con los resultados de otras investigaciones que abordan la misma problemática, encontrando la posición esquiva de padres y representantes a conformar y participar en dichos cuerpos organizativos.

Del mismo modo, en Perú, Ochoa (2018) llevó a cabo una investigación sobre la participación de los padres de familia como corresponsables de la educación de sus hijos en una institución educativa del Callao. La misma, se desarrolló en el paradigma positivista con enfoque metodológico cuantitativo bajo una investigación de campo de carácter descriptiva con diseño no experimental transaccional descriptivo. La unidad de análisis estuvo enfocada en la Institución Educativa № 5041 de Carmen de la Legua ubicada en el Callao. La muestra fue no probabilística conformada por 110 padres de familia de dicha institución.

Para la recolección de datos, se utilizó un instrumento tipo cuestionario estructurado estandarizado para padres de familias de Valdés, adaptado por la investigadora y sometido a juicio de tres (3) expertos y calculada su confiabilidad con valor de 0,85 que lo describe altamente confiable. Al procesar los datos, se obtuvo como resultado, la predominancia del nivel regular con respecto a la participación de los padres de familias; niveles muy altos en la dimensión comunicación y aprendizaje en casa; caso contrario en las dimensiones cooperación en la escuela, comunidad y voluntariado.

Los trabajos antes presentados, son de gran relevancia para esta investigación por cuanto devela la postura que mantienen los padres y representantes ante los consejos escolares que dificulta la participación social en los escenarios educativos que los hace complejos, premisas que coinciden con las intencionalidades de este trabajo que busca asumir un punto de vista filosófico para explicar y clarificar el conocimiento acerca del pensamiento de las acciones humanas en ese contexto. 


\section{Teoría crítica y complejidad en la participación social}

Con el fin de dar fundamento epistémico a la aproximación teórica, fue pertinente basar sus fundamentos en la Teoría Crítica y Complejidad en la Participación Social. En ese sentido, el hombre desde su origen ha estado constantemente en la búsqueda de respuestas de su propia existencia y del papel que le toca desempeñar dentro de la sociedad en la que interactúa, es así como surgen una serie de teorías que apuntan hacia su explicación, entre las cuales se destaca la teoría crítica de Habermas (1991), orientada al encuentro de una transformación social, cuyo interés reside en la emancipación del sujeto en un contexto democrático; teniendo presente la naturaleza política de todo proceso social, y por ende del quehacer educativo, de ahí que se oponga a la racionalidad instrumental.

Dicha teoría, plantea que la única forma de transformación social y emancipación del sujeto se da en un contexto democrático, por ser el único mecanismo que permite la participación activa del individuo en la toma de decisiones libremente de manera individual y colectiva. Éstas premisas, están estrechamente vinculada al objetivo general de esta aproximación teórica debido a que se centran en la persona como actor fundamental del proceso de cambio de escenarios educativos complejos. Al respecto, Habermas (1991) sostiene que la acción pedagógica está definida como una práctica social, dialógica, histórica y contextual proceso complejo, incierto, dinámico, ecológico, que se configura en las construcciones de significado de los participantes, con fuerte componente ético, expresado en relaciones dialógicas establecidas en una dinámica cuyo eje son los valores democráticos.

Con la teoría antes descrita, se puede abordar un conjunto de situaciones continuamente revisables y modificables puesto que, la realidad social así lo amerita, por ser cambiante en el tiempo y espacio, ya que es evidente que el hombre es el agente ejecutor de todas las acciones que se llevan a cabo por cuanto tiene la capacidad de aprender individual y colectivamente, como también de integrarse en la realidad que le corresponde vivir en tiempo y espacio. Todos estos elementos tienen que ver con la construcción teórica que se propone con la realización de este estudio.

En cuanto al pensamiento complejo, Morín (1995) sostiene que existen diversos niveles de realidad y que toda realidad es un sistema por el hecho de estar en relación con su contexto. Es fundamentalmente, un pensamiento que relaciona y que es capaz de producir sucesivas religaciones, razón por la cual el objeto de conocimiento debe ser estudiado a su vez en relación con lo que lo circunda. Es pues, un pensamiento abierto que rompe con el cuarto precepto lógico o regla para la dirección del espíritu propuesta por descartes: hacer en todo enumeraciones tan completas y revisiones tan generales que estuviera seguro de no omitir nada (Descartes 1981).

Asimismo, plantea Morin (1995) en su método de la complejidad, que hay que dejar de lado dos ilusiones heredadas del pensamiento positivista, la primera, considerar que la complejidad conduce a la eliminación de la simplicidad, porque, al contrario, el pensamiento complejo une, integra, va a la búsqueda de conexiones. En este sentido, rechaza el reduccionismo, el cual considera la descomposición del todo en sus partes para conocerlo. Pero también, hay que estar alerta para no sobrevenir en la segunda ilusión, que es creer que el pensamiento complejo es completo, porque sería caer en la totalidad como un absoluto, no se debe olvidar que el conocimiento es inacabado e incompleto.

Frente a esta visión Cartesiana que busca la integridad por la vía de la exhaustividad, el pensamiento complejo plantea la heterogeneidad, la interacción, el azar y se rige por éstos y por los siete principios guía para un pensamiento vinculante, resumidos por Morín (1995) en: principio sistémico u organizativo, que une el conocimiento de las partes con el conocimiento del todo; principio holográfico de las organizaciones complejas, que no es más que la parte está en todo, pero también el todo está en cada parte; principio del bucle retroactivo o retroalimentación, referido a que la causa actúa sobre el efecto y el efecto sobre la causa; principio del bucle recursivo, donde los productos y los efectos son en sí mismos productores y causantes de los que se produce. 
Del mismo modo están, el principio de autonomía/dependencia que sostiene que los seres vivos gastan energía en mantener su autonomía, por consiguiente necesitan encontrarla en su medio ambiente, en consecuencia, su autonomía es inseparable de esa dependencia; principio dialógico entre el orden, el desorden y la organización a través de innumerables interacciones en constante acción; principio de reintroducción del que conoce en todo conocimiento, que es una reconstrucción/traducción que hace una mente/cerebro en una cultura y un tiempo determinados.

Ese pensamiento dirigido por esos principios, se corresponde con una visión compleja de la realidad y se considera como el sustrato fundamental del enfoque y del método transdisciplinario con los cuales es posible abordar e investigar la realidad en toda su complejidad. Pues bien, si la transdisciplinariedad llegase a establecerse definitivamente en el plano del conocimiento, debería en consecuencia, extrapolarse a la educación por ser dichos procesos complejos que ocurren a lo largo de la vida. En consecuencia, la participación social, objetivo principal del estudio, se fundamentan en éstos principios, por cuanto en su conjunto forman parte del análisis de la realidad.

Aunado a ello, Morín (1995) plantea que la complejidad comienza a aparecer cuando uno se pregunta por el sentido de la historia y se da cuenta que el único sentido es el que se va construyendo conforme se hace historia, a lo que se agrega la capacidad de inventiva y creatividad del ser humano, de lo cual se desprende que no es posible predecir el futuro socio histórico, porque una continua creación hace imposible la predicción. Desde ese punto de vista, se puede decir que los problemas actuales no pueden ser entendidos aisladamente por estar interconectados y ser interdependientes, dado que desde la complejidad hay un llamado a pensar de modo ecologizante; esto es, considerar al objeto estudiado en vez de aislarlo en y por su relación eco organizadora con su entorno.

Para complementar la perspectiva epistémica, se consideró importante tomar en cuenta algunos preceptos de la teoría humanista, cuya perspectiva centra su interés por conocer al individuo y las potencialidades que tiene como posibilidad de desarrollar las cualidades más elevadas que posee, que le van a permitir entender lo que es el mundo y lo que es el hombre, es decir, su posición paradigmática y su capacidad para aprender de manera individual y colectiva, así como también en la de sumergirse en la realidad tiempo y espacio que le corresponde vivir y transformar sus estructuras. Es un hacer y rehacer constante a través de la reflexión y la participación en escenarios educativos, considerando éste como el instrumento para el logro de los grandes cambios que se deben dar dentro de la sociedad.

Adicional a lo antes planteado, el humanismo implica la creencia en la unidad de la raza humana y en el potencial del hombre para perfeccionarse a través de su propio esfuerzo. Es por ello, que, en todas sus vertientes, la idea fundamental es la responsabilidad del hombre para crear condiciones de vida valederas, que puedan ser llamadas humanas, compartiendo experiencias, responsabilidades y valores para que puedan unir su trabajo mental con el manual y encontrar así su felicidad de ser y en colaboración con otros, tal como lo refiere Fernández (1979).

\section{Reforma sin participación activa del ciudadano}

En Venezuela, con la llegada de Hugo Chávez Frías a la presidencia del país, se hizo la reforma a la Constitución de la República Bolivariana de Venezuela (CRBV) dando paso a la aprobada en 1999 con la promesa de un cambio a favor de la participación social. Sin embargo, el camino transitado en éstos últimos veinte años fue hacia un cambio opuesto al país democrático que históricamente ha sido siempre, de manera severa y radical contra puesta a los fundamentos teóricos, filosóficos y políticos de cualquier democracia.

Es por ello que, sin ánimo de fijar un juicio valorativo sobre los aspectos que se plantearon en dicha reforma, es significativo señalar que la situación actual en cuanto a participación es contradictoria sobre lo que se dice en el texto constitucional y lo que realmente se permite en función de lograr una mayor 
participación del ciudadano y ciudadana en las decisiones sobre materia que los afectan directamente, como la educación, debido a que en la práctica éstas no se corresponden con los preceptos democráticos universales allí plasmados.

Prueba de ello, es la conducción del país por parte del gobierno hacia la construcción de una sociedad socialista, donde el Estado es quien dicta lo que se debe hacer en todos y cada uno de los poderes constituidos. De esa manera, educar a los ciudadanos para participar en la construcción de la sociedad no es tarea fácil, dado que allí no hay cabida a una sociedad participativa que se pueda imaginar libremente. Esto, a pesar de contradecir al artículo 62 de la CRBV (1999) que establece que los ciudadanos y ciudadanas tienen el derecho de participar libremente en los asuntos públicos, directamente o por medio de sus representantes elegidos o elegidas. Por consiguiente, es obligación del Estado y deber de la sociedad facilitar la generación de las condiciones más favorables para su práctica.

De igual manera, está el artículo 3 de la CRBV (1999) que refiere que el Estado tiene como fines esenciales: la defensa y el desarrollo de la persona; el respeto a su dignidad; el ejercicio democrático de la voluntad popular; y, la construcción de una sociedad justa amante de la paz, entre otras declaraciones. Sin embargo, a pesar de lo antes planteado, en la práctica no se permite la libertad de pensar con autonomía, ni el desempeño libre de los individuos y con uso de razón; debido a que es el Estado quien dice y dicta las pautas de comportamiento.

Lo antes planteado, hace difícil enmarcar desde la perspectiva legal la participación social de la comunidad educativa en Venezuela en un contexto mucho más complejo hoy día por las circunstancias políticas aludidas; y en donde cada día se puede apreciar la radicalización de represión por parte del Estado que ha logrado paulatinamente la destrucción de todo el sistema educativo. No obstante, esto no ha logrado apagar la luz del interés por la participación de los ciudadanos; de allí el interés de esta investigación encausada a generar una aproximación teórica acerca de la participación social de la comunidad educativa en escenarios complejos de educación media en Venezuela.

\section{Metodología}

La metodología empleada, para la realización de esta investigación estuvo orientada en el paradigma interpretativo con un enfoque metodológico cualitativo, epistémico fenomenológico y fenomenológico hermenéutico. Esto con la pretensión de conocer el cómo los actores perciben o construyen en su contexto particular, en su vida cotidiana y dentro de las relaciones ínter subjetivas.

En cuanto al paradigma interpretativo, Pérez (2002) señala que su propósito es develar el significado de las formas particulares de ver la vida social mediante el enlace de estructuras de significado subjetivo que guían las maneras de actuar de las personas. Para ello, es necesario el enfoque metodológico cualitativo que de acuerdo con Sandín (2003) está dirigido a la comprensión de fenómenos sociales, así como al descubrimiento de un corpus de conocimientos. Por su parte, Martínez (2006) agrega que ese paradigma busca detallar las realidades y su dinámica para dar razón de su actuación y revelaciones.

Dicho paradigma y enfoque metodológico, permitieron centrarse en develar e interpretar la realidad desde el pensamiento, creencias, sentires y otras características no observables ni susceptibles de experimentación y/o verificación, tales como significados e interpretaciones construidas por los informantes clave, que ayudaron a emerger los constructos teóricos relacionados con la participación social de la comunidad educativa en escenarios complejos de educación media en Venezuela, para poder generar la aproximación teórica.

En cuanto al enfoque epistémico fenomenológico, trata de descubrir las estructuras fundamentales de la conciencia; no es tanto descubrir el fenómeno, sino hallar en él la esencia válida y útil científicamente. Su atención está puesta en el fenómeno mismo, en lo que presenta y revela la propia conciencia y del 
modo que lo hace con toda su concreción y particularidad. Esto no sólo tiene su estructura y regularidad, sino una lógica que es anterior a otra lógica, tal como lo plantea Husserl, citado en Martínez (2006).

En cuanto al método fenomenológico hermenéutico, Heidegger (2005) sostiene que éste se sustenta existencialmente en la interpretación y comprensión, siendo ésta una de las posibilidades del ser. Se interesa, además, en develar la existencia humana, considerando tanto la experiencia como el significado del ser a través de la descripción que significa restituir la evidencia a la experiencia desde la viva voz de quienes la viven. Según el precitado autor, dicho método busca comprender las realidades vivenciales de otras personas respetando absolutamente la relación que hacen éstas de sus propias vivencias.

\section{Unidad de análisis e informantes clave}

La unidad de análisis, se refiere al escenario seleccionado para el desarrollo de la investigación. De acuerdo con Teppa (2012), es el contexto al cual el observador tiene fácil acceso, consigue establecer una buena relación inmediata con los sujetos que participan en el estudio y por consiguiente puede recoger directamente de ellos la información relacionada con su trabajo. De acuerdo con la precitada autora, la unidad de análisis más recomendable por su asequibilidad es el lugar donde labora el investigador. Por tal motivo, se seleccionó un liceo del municipio Crespo, Estado Lara (Venezuela).

Esa particularidad, favoreció la espontaneidad de las conversaciones y la seguridad para expresar sus opiniones, pensamientos y sentires sobre el significado que le atribuyen al fenómeno en estudio; sin desperdiciar la observación de su lenguaje gestual y corporal a la hora de expresar sus discursos provocados por el investigador. Todo ello, permitió disfrutar de la narración del fenómeno social abordado a través de las percepciones de la realidad de quienes la han vivido.

En ese sentido, se seleccionó como informantes: un (1) director, un (1) profesor, un (1) representante, por ser clave y privilegiados por su capacidad informativa del fenómeno en estudio desde la perspectiva del rol que cada uno de ellos ejerce dentro de la institución escolar. Al respecto, Hernández, Fernández y Baptista (2010) afirman que, en el enfoque metodológico cualitativo, no existe parámetro para el tamaño de la muestra porque ello va en contra de la naturaleza de la indagación cualitativa. Es decir, la decisión del número de informantes queda a criterio del investigador. El principal factor a tomar en cuenta para ello, es que los seleccionados proporcionen un sentido de comprensión profunda del problema de investigación; allí no importa la cantidad, incluso puede tratarse de una sola unidad de análisis como en los estudios de caso, dado que éstos no representan a una población.

Además, esta información se interpretó después en el marco de la situación que la generó, y para lograrlo hubo que compenetrarse con los participantes detallando cuanto fue necesario en el proceso de investigación. Por con siguiente, se fijó un grupo de criterios, que le dieron una imagen global a los informantes clave, quienes fueron elegidos de manera intencionada en atención a lo expresado por Martínez (2006), quien sugiere que, en las investigaciones con enfoque metodológico cualitativo, éstos no podrán ser elementos aleatorios descontextualizados, sino por un todo sistémico con vida propia, como es una persona, una institución, una etnia o grupo social. Por consiguiente, la escogencia respondió al perfil característico relacionado con el objeto de estudio.

\section{Recolección y procesamiento de la información}

De acuerdo con la postura metodológica se utilizó la entrevista semi estructurada como técnica en los términos expresados por Hernández, Fernández y Baptista (2010). Cónsono con la entrevista como instrumento se utilizó una guía simple que ayudó a orientar la conversación con preguntas que permitieron al entrevistador la libertad de introducir premisas adicionales que le proporcionen la información requerida sobre el tema tratado. Ésta resultó de gran ayuda para que los informantes clave aportaran información valiosa sobre el fenómeno. 
Luego de obtener el discurso pormenorizado de cada uno de los informantes clave, se transcribió de manera textual y se extrajeron posteriormente las categorías, sub categorías, descriptores, que ayudaron a develar los constructos teóricos relacionados con la participación social en el marco del pensamiento complejo y transdisciplinario en escenarios educativos y así generar una aproximación teórica. Para encontrar el punto de saturación, se establecieron nuevos encuentros con los informantes para aclarar ideas, se hicieron nuevas grabaciones hasta obtener la información requerida para llevar a cabo las intencionalidades de esta investigación.

La información aportada por los informantes clave, se procesó a través de los procedimientos recomendados para la aplicación del método fenomenológico hermenéutico planteados por Pérez (2002) que apuntan a que la información recogida se debe organizar en unidades más sencillas con la finalidad de identificar uniformidades en ellas que permitan al investigador evidenciar qué es importante y qué aporta al estudio. Para ello, se realizó de manera repetida la lectura de las entrevistas que luego se transcribieron (Anexo A) para poder descomponer la información obtenida, donde emergieron las categorías, sub categorías y descriptores de la investigación.

Para llevar a cabo el proceso anterior, fue indispensable que detallara de manera minuciosa, línea por línea para poder detectar las categorías, sub categorías y descriptores inmersos en los significados provenientes de las entrevistas de cada uno de los informantes clave, lo que demandó asignarle un código a cada uno de los elementos para mejor comprensión de los mismos.

\section{Codificación, categorización, estructuración e integración de la información}

La codificación transitó por lo propuesto por González y Hernández (2011) y que demandó a demarcar el espacio físico del texto (una línea o un bloque) con varios colores, números, letras u otros símbolos, para indicar la ubicación de una o varias categorías o subcategorías. En ese sentido, y dentro de lo sugerido por los autores antes mencionados, se decidió trabajar con códigos alfanuméricos para referirme a los informantes, con la intención de resguardar la identidad y respetar su anonimato, los identifiqué como IC1D (Director), IC2P (Profesor), IC3R (Representante).

Para las categorías, sub categorías y descriptores, se utilizó código alfabético: Participación Social (PS); Escenarios Educativos Complejos (EEC). Para identificar si los elementos encontrados se refieren a una categoría, sub categoría o a un descriptor, se asignó un código alfabético en mayúscula: C (categoría); SC (sub categoría); D (descriptor). Eso, ayudó a fragmentar los contenidos en unidades temáticas que expresaron una idea o concepto central por lo que representan una categoría, de acuerdo con la postura de Martínez (2006) quien sostiene que categorizar es hacer una organización a través de la conceptualización y codificación representada en un término o palabra, la idea central de cada unidad temática obtenida de la reducción sistemática de la información; es decir, reducir la información en sus componentes más importantes.

En cuanto a la estructuración de la información, se realizó de manera artesanal como procedimiento metódico con actitud creativa y reflexiva para poder interpretar la realidad percibida por quienes viven el fenómeno. Del mismo modo, se impregnó de manera subjetiva en un proceso dialógico desde la lógica fenomenológica al momento de interpretar la información; asumiendo una postura como investigador. De esta manera, la interpretación no es ninguna descripción emanada de un espectador neutral, sino más bien, un evento dialógico en el que los interlocutores se ponen en juego por igual y del que salen modificados (Crespo, 2018).

\section{Contrastación y triangulación de la información}

La primera contrastación que se realizó, fue cuando se sometió a confrontación la información que se obtuvo de las entrevistas con los informantes clave en un retorno sistemático que permitió revisar con ellos lo recogido para saber si en realidad fue lo que quisieron expresar en torno al fenómeno, tal como 
lo sugieren Taylor y Bogan (1994); quienes expresan que es la devolución de la información a los informantes clave, para validar, si se logró comprender y reconstruir su realidad.

Luego, en el proceso heurístico, la contrastación se hizo con apoyo de la triangulación de la información aportada por los informantes clave, fuentes teóricas y patrimonio intelectual del investigador como docente en el contexto escogido. Esta triangulación transcurrió a la luz de la contrastación entre diferentes fuentes de información aportadas por personas, instrumentos, documentos o combinación de todos (Kemmis, 2006; Rojas, 2007).

Por consiguiente, la comprensión del fenómeno estudiado la hice desde los intereses del estudio en una especie de combinación entre las informaciones del IC1D, ICP2, IC3R, los aspectos teóricos y conceptuales que orientan las categorías emergentes y la postura como investigador. Dicho ensamble de informaciones, sirvió para discernir la interpretación en la búsqueda de la comprensión del fenómeno estudiado desde una perspectiva ontológica, epistemológica y metodológica fenomenológica que dio paso a la generación de la aproximación teórica de esta investigación.

\section{Integración de los hallazgos emergentes}

La integración de los hallazgos emergentes del fenómeno estudiado en la investigación, se realizó con la incorporación de los testimonios de los informantes clave, obtenida a través del método fenomenológico hermenéutico, que permitió acercarse al conocimiento ontológico de la aproximación teórica de esta investigación. Al respecto, Teppa (2012) la llama proceso de análisis que sigue al trabajo de campo o recolección de información, que genera como resultado, la elaboración de un producto, bien sea una teoría, un modelo o redacción de un informe sobre reflexiones finales.

En ese sentido, fue un asunto complejo que exigió en todo momento comprensión para lograr la reflexión en el transcurso del trabajo, por ser un proceso cíclico que demandó descomponer en sus partes más básicas el discurso de los informantes clave; reducir hasta llegar a sus principales elementos e ideas más relevantes, en atención a lo sugerido por Teppa (2012), quien además agrega que se debe tomar en cuenta su esencia, las concepciones de mayor significado para la investigación, cuya integración cognitiva ayuda a construir la teoría, como en efecto ocurrió en este estudio, dando así elementos necesarios para alcanzar la intencionalidad investigativa y de esa forma, generar la aproximación.

\section{Resultados}

Los resultados de la investigación, se desarrollaron en dos (2) vertientes: la ontológica aportada por los informantes clave y la epistemológica producto de la revisión de lo que han escrito los autores sobre el fenómeno, que luego fueron integradas para obtener los hallazgos y reflexiones finales que permitió generar la Aproximación Teórica acerca de la participación social en escenarios educativos complejos, la cual se espera contribuya al fortalecimiento de la condición humana dentro de la sociedad tan cambiante, donde debe prevalecer la democracia, justicia, valores de igualdad, tolerancia y sobre todo la paz que tanto se anhela.

Para interpretar la participación social desde las voces de los actores significantes en los contextos educativos complejos se procedió a la conformación de unidades de análisis de cada uno de ellos, tal como se presentan a continuación: 
Tabla 1

Unidades descriptoras del IC1D en el estudio

Unidades Descriptoras

- Se convoca a los representantes para que participen en las diferentes actividades programadas, sean éstas de tipo cultural o académicas.

- Su asistencia a la institución es bastante difícil, ya que manifiestan vivir lejos; sin embargo, siempre están pendientes a cualquier llamado.

- Las convocatorias siempre se pasan por escrito y con anticipación.

- Siempre las convocatorias se hacen hasta tres veces para poder hacer las asambleas. No se sabe que pasa, se tendrán que tomar medidas al respecto en base al reglamento interno.

- Los representantes en su mayoría son madres, y por lo general, trabajan en casas de familia y no le dan permiso.

- La comunicación que tenemos con los representantes, la hacemos regularmente en la puerta de la escuela cuando llevan a sus niños(as).

- La comunicación de los directivos de ambos turnos (mañana y tarde), se hace mediante un cuaderno de notas donde se colocan todas las incidencias.

- Los representantes se sienten muy bien, cuando se les comunica que hay actividades culturales, fiestas por grados, así si asisten.

- Se elaboran avisos y pancartas para comunicarles a los representantes de cualquier actividad que se vaya a realizar en la institución, además de anotarles a sus hijos(as) la invitación en sus cuadernos.

La efectiva participación entre los miembros de una institución genera consenso y compromiso, más allá del grupo central de actores; sino también con la comunidad en general, pues se consigue transmitir y planificar los proyectos con un enfoque estratégico y una visión compartida, cuyo resultado sea el compromiso y la voluntad de actuar. Al efectuar el análisis de la sub comunicación, definida como el conjunto de intercambio de información entre directivos, docentes, directivos de la asociación de padres y representantes. En las respuestas expresadas por los directivos, se infiere que, en los procesos comunicativos establecidos con los diferentes actores comunitarios, ya sea de forma oral, en las puertas del plantel o en forma escrita, les permite mantener intercambios irrelevantes y no pertinentes en el proceso de reforma que está planteado por el Ministerio del Poder Popular para la Educación. 
Tabla 2

Unidades descriptoras del IC2P en el estudio

Unidades Descriptoras

- Casi nunca la comunidad llama a los docentes a reuniones, regularmente somos los docentes que le convocamos para que ellos participen.

- Son muy pocos los representantes que participan en las actividades de la escuela.

- Los representantes sólo se interesan en ir a la escuela a buscar las notas a fin de año para ver si el muchacho pasó de grado.

- El canal inmediato para comunicarnos con el representante es el alumno.

- La comunicación: docente y representante se hace en el momento de entrada a clases.

- La comunicación entre docentes y representantes se hace por escrito.

- Aquí falla mucho la comunicación entre directivos y docentes.

- Cuando llegan comunicaciones para talleres, reuniones sindicales, etc., no se asiste para que los niños no pierdan clases.

- Siempre nos comunicamos lo que está ocurriendo en el plantel.

- Algunas veces hay informaciones que no las comunican a tiempo.

De acuerdo con las respuestas expresadas por el IC2P, indica que son ellos quienes promueven la comunicación dialógica y participación, la cual permite decidir las estrategias de acción, planificación y ejecución de proyectos educativos, aun cuando los representantes no acudan al llamado. Situación que muestra la buena disposición del IC2P a la participación, ya que sus expresiones manifiestan compromiso con la institución, así como también la iniciativa de promover la asistencia de los representantes cuando llevan a cabo actividades donde involucran a los miembros de la comunidad educativa, a pesar de expresar que la comunicación no es la más óptima.

Tabla 3

Unidades descriptoras del IC3R en el estudio

Unidades Descriptoras

- Creo que la participación es asistir e intervenir en cualquier cosa que se presente en la escuela.

- Regularmente no asisto a las reuniones porque no me gusta involucrarme en los problemas.

- La asistencia de los demás representantes a veces es precaria; todo depende de lo que se vaya a tratar en la reunión; si es porque los niños no tienen maestra, porque está de permiso y están perdiendo clase, así sí asisten.

- La mayoría que asisten a las reuniones son mujeres, ya que los hombres trabajan y no pueden ir.

- Cuando me llegan las invitaciones a las asambleas, no me gusta asistir y menos participar porque se ríen de lo que uno dice y eso me hace sentir muy incómodo, hasta el extremo de perder mi compostura, es lo que le dicen los representantes a uno.

- Es una eterna pelea con los maestros para que asistamos y ellos no entienden que tenemos problemas para asistir, que tenemos que trabajar.

- Asisto cuando me invitan para la entrega de boletas y para saber cómo van saliendo los hijos.

- Asisto a las reuniones cuando es muy urgente y me lo mandan a decir por escrito.

- Cuando el docente me manda a razones con su hijo, estoy pendiente. 
Los resultados de las entrevistas realizadas reflejan el escaso conocimiento de los representantes en relación al concepto de participación, determinando esto la poca asistencia a las diferentes actividades a las cuales se les invita. Igualmente, se evidencia que su asistencia está condicionada por el grado de importancia que tenga la reunión a la cual se les invitó y que el estudiante le recuerde de la reunión. Otro de los elementos importantes para destacar, es la apatía de asistir a cualquier tipo de actividad o reunión en el centro educativo. El interés que lo mueve a asistir, es la buscar la boleta de calificaciones de su representado, cuando se le es obligado de manera escrita o si el asunto a tratar es la pérdida de clase de su hijo cuando el docente está de permiso. Todo lo cual, desdibuja el concepto de la participación en escenarios educativos.

Por otra parte, para develar los constructos teóricos relacionados con la participación social en escenarios educativos complejos, se hizo la integración de la información aportada por los informantes clave, para confrontarla con las premisas epistémicas seleccionadas sobre el fenómeno para obtener de esa manera la síntesis interpretativa. Para una mejor comprensión, se utilizó el siguiente cuadro comparativo:

Tabla 4

Resultados integrados según cada una de las sub categorías

Sub Categorías Resultados Integrados

Suele ser improvisada, espontánea e intermitente, en cuanto que los padres y representantes acuden diariamente para enterarse del proceso educativo protagonizado por sus representados. La asistencia a reuniones o asambleas a veces es obstaculizada por la falta de respeto exhibida por los directivos al expresar los padres y representantes sus ideas. También está supeditada al

Asistencia tiempo libre y a intereses personales. Los docentes la ejercitan y la promueven en el resto del personal al involucrarlos en los proyectos educativos.

La información relacionada con las asociaciones educativas llega a los actores escolares por los canales regulares, sin embargo, se logra; la informalidad y

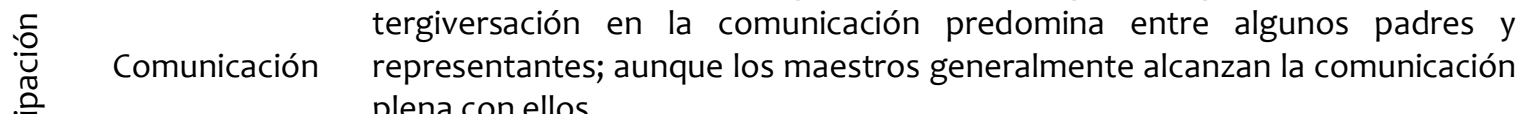
plena con ellos.

Supeditada a los estados anímicos de los participantes, desconocimiento del término, reflejado en la parquedad de las respuestas. Los docentes en su mayoría logran la interacción con los representantes en la planificación y Interacción ejecución de los proyectos educativos integrales comunitarios (PEIC) y otros se justifican alegando exceso de trabajo.

Improvisada, restringida en casos particulares, dependiendo del tiempo libre y de la posibilidad individual, desplegada ante las emergencias presentadas y las necesidades de autogestión de la institución y practicada por los docentes en su mayoría; cuando colaboran y participan activamente en

Colaboración aspectos relevantes de la educación de los estudiantes, demostrando sensibilidad y comprensión hacia los demás.

La misma se desarrolla de forma espontánea o cotidiana o en función de las actividades planificadas por los docentes y cuando redunda en un beneficio inmediato; la asociación civil de padres y representantes es la abanderada en

Cooperación cuanto que cumple a cabalidad con sus objetivos; y finalmente, carencia de proyectos planificados en conjunto merma el dinamismo de la actividad cooperativa.

Los cambios se han evidenciado a nivel de la planificación y evaluación de los proyectos educativos planificados por los docentes (PEIC), y ejecutados por 


\begin{tabular}{cc}
\hline $\begin{array}{c}\text { Promoción de } \\
\text { cambios }\end{array}$ & $\begin{array}{c}\text { los alumnos, padres y representantes en el plazo pertinente, mas no en los } \\
\text { directivos por el excesivo trabajo administrativo y la carencia de la planificación } \\
\text { en los proyectos de aprendizaje (PA). }\end{array}$ \\
\hline Participación & $\begin{array}{c}\text { Es relevante la participación de los miembros de la comunidad cuando está } \\
\text { relacionada con el bienestar personal de los educandos o referidas al proceso } \\
\text { de enseñanza. Es importante señalar que el docente transmite su apreciación } \\
\text { de la importancia y responsabilidad de todos. Igualmente mantiene y asume su } \\
\text { compromiso en la mayoría de las actividades planificadas }\end{array}$ \\
\hline Compromiso & $\begin{array}{c}\text { El compromiso de todos los actores escolares con el proceso educativo y el } \\
\text { asumir la responsabilidad en su relación con la familia y el entorno, tiene que } \\
\text { ser producto de un trabajo personal de auto crecimiento y maduración. Pero la } \\
\text { carencia de proyectos institucionales en donde se realicen acciones concretas } \\
\text { que garanticen el compromiso de la comunidad escolar, no promueve la } \\
\text { apertura a este tipo de comportamiento. }\end{array}$ \\
\hline
\end{tabular}

\section{Discusión}

A la luz del análisis de cada una de las categorías y plasmados los resultados integrados se puede decir que la subcategoría participación, develó que los actores de la comunidad escolar asisten a la institución y a las reuniones dependiendo de la promoción, motivación y convocatoria. En tal sentido, Argyris y Shon (2003) señalan que generalmente ese es el grado de participación que se logra normalmente con los miembros comunitarios. Mencionan, también, que, en ámbito organizacional, el aprendizaje se conoce como el cambio de comportamiento manifestado en la conducta colectiva de sus actores, quienes se identifican no sólo con su función particular, sino con el todo de la institución, asumiendo y compartiendo el compromiso de aprender a pensar sistemáticamente, en términos de entender y asumir las consecuencias de sus actos y el de las múltiples fuerzas e interconexiones que afecten la organización.

Con respecto a la subcategoría comunicación, son los docentes quienes establecen con mayor efectividad este proceso, pues mantienen, según la posición de Argyris y Shon (2003) una comunicación dialógica con sus colegas y con padres y representantes, incentivándolos a la participación activa en la planificación y ejecución de los proyectos educativos.

De igual manera, el análisis de la subcategoría integración presentó las mismas características: los docentes son los que promueven la interacción y logran generalmente con los padres y representantes la articulación objetivos, metas y estrategias comunes que incidan en las opiniones y decisiones que se tomen frente a los problemas que rodean el proceso educativo. Al respecto, Hernández (1994), señala que es característico de los grupos que se están iniciando, que están en proceso de consolidación y tras la búsqueda de alternativas de solución a la problemática existente.

En cuanto a la colaboración, se implementa en todos los actores del contexto escolar cuando hay situaciones de emergencia (enfermedades, muertes, accidentes, entre otros) o cuando hay que solventar problemas del plantel a través de la autogestión. Asimismo, son los profesores los que canalizan la misma, y según el precitado autor, es la comunidad quien produce la negociación entre los distintos actores y cada cual actúa a partir de sus propios recursos y demanda de los otros que cumplan con lo acordado. De esta manera, los docentes la ponen de manifiesto cuando comparten, colaboran y contribuyen con todos los miembros de la comunidad escolar.

El análisis de la subcategoría cooperación, dio como resultado que la mayoría de los miembros del contexto escolar la practican desde la diversidad de roles que les corresponde desempeñar: padres y representantes; docentes; directivos, están comprometidos con el buen funcionamiento de la institución y asumen con libertad sus funciones. En ese sentido, es oportuno mencionar a Gómez del Campo (2004), 
quienes señalan que el ser humano es eminentemente social y cooperativo, dimensión que lo lleva a insertarse en la realidad social donde labora y busca respuesta a los problemas planteados.

En cuanto a la subcategoría promoción de cambios, es preciso señalar que éstos en el sector educativo dependen de los organismos rectores del sistema con el propósito de mejorarlo, integrarlo o aumentar su eficacia, tal como lo expresa Lanz (2006). Por consiguiente, las estrategias didácticas, deben planificarse a partir de las demandas e intereses de los docentes, padres, representantes y la realidad social, económica y cultural que circunda la escuela, facilitando la participación de todos para decidir en forma consensuada los objetivos de interés para y con el educando. Otros de los cambios, fue la elección de la asociación civil de padres y representantes en el primer trimestre del año escolar; actividad que se cumplió con la asistencia de todos los representantes y docentes de la institución.

Por su parte, la participación es relevante en los miembros de la comunidad cuando está relacionada con el bienestar personal de los educandos o referidas al proceso de enseñanza. En ese sentido, el docente transmite su apreciación de la importancia y responsabilidad de todos. Igualmente, mantiene y asume su compromiso en la mayoría de las actividades planificadas. Para finalizar, al revisar la integración todos los actores escolares del proceso educativo asumen la responsabilidad en su relación con la familia y el entorno, producto de un trabajo personal de auto crecimiento y maduración. En contraposición a ello, se observa la carencia de proyectos institucionales con acciones concretas que garanticen el compromiso de la comunidad escolar; es decir, éstos no promueven la apertura a ese tipo de comportamiento.

El análisis de cada una de las subcategorías, reflejaron el grado de participación de los miembros de la comunidad educativa en el centro escolar donde se llevó acabo el levantamiento de la información, cuyo punto significante está en el proceso participativo desarrollado por padres y representantes caracterizado por ser continuo, cotidiano y constante, pues ellos acuden a diario y masivamente a la institución motivados por el bienestar de sus representados.

La actitud sostenida por los mencionados actores del contexto escolar en estudio constituye una fortaleza, ya que, en su mayoría, son personas preocupadas por sus representados y su proceso educativo. Los docentes ante el proceso en referencia, observan el desarrollo de una dinámica integrada y continua que estimulan, a través de estrategias implementadas en los proyectos educativos, la cual permite y genera cambios que conllevan a la participación real y efectiva de la comunidad escolar. La parte directiva, acredita la actuación institucional y revelan que son los llamados a establecer pautas para efectuarla de manera efectiva y permanente en la programación de proyectos educativos integrales.

En atención a lo antes planteado, éstos manifestaron su compromiso para el logro de los objetivos comunes, a fin de mejorar la calidad de vida, tanto escolar como comunitaria, en función de la transformación social planteada en el contexto educativo y atendiendo a las necesidades del grupo. Esto, a través de la disposición, esfuerzo, ayuda mutua y el trabajo en grupo; indispensables para resolver en una comunidad y la escuela problemas sociales y materiales de manera organizada.

\section{Referencias}

Argyris, C. y Shón, D. (2003). Conocimiento para la Acción. Una guía para superar obstáculos del cambio en la organización. Buenos Aires - Barcelona - México: Granica S.A.

Bloom, E. (2010). PEC y el Banco Mundial. Educare. Renovación Educativa. Recuperado de https://issuu.com/pecnacional/docs/renovacionespecial

Constitución. (1999). Gaceta Oficial de la República Bolivariana de Venezuela Nº 36.850 del martes 14 de diciembre de 1999. Caracas: Presidencia de la República de Venezuela.

Crespo, R. (2018). Visión Fenoménica de los Gerentes Farmacéuticos Acerca del Liderazgo en Tiempos de Crisis (Tesis de doctorado). Universidad Fermín Toro. Cabudare, estado Lara.

Descartes, R. (1981). Discurso del Método. Castellón: Los libros de Plon. 
Díaz, J., Santisteban, A., y Cascajero, A. (2013). Medios de comunicación y pensamiento crítico. Nuevas formas de interacción social. España: Editorial Universidad de Alcalá.

Fernández (1979). Las Estrategias Cuantitativas y la Investigación en la Enseñanza. UCV - CONICIT. Primer encuentro de Creatividad 90. Valencia, Venezuela.

Gómez del Campo (2004). Formación del Psicólogo Profesional desde una Perspectiva Humanista con Énfasis Comunitario. Compilado por Lafarga y Gómez: Desarrollo del Potencial Humano. México: Trillas.

González, G., y Hernández, T. (2011). Interpretación de la Evidencia Cualitativa. Más allá del Glater. Barquisimeto, Venezuela: Ediciones Gema, C. A.

Habermas, J. (1991). Teoría de la Acción Comunicativa (Volumen I y II). Madrid: Taurus.

Habermas, J. (2000). Teoría y Praxis. Estudio de Filosofía Social. España: Editorial Tecnos, S.A.

Heidegger, M. (2005). Ser y Tiempo. México: Fondo de Cultura Económica.

Hernández, E. (1994). Elementos que Facilitan o Dificultan el Surgimiento de un Liderazgo Comunitario. Guadalajara, México: Ediciones de la Universidad de Guadalajara.

Hernández S., Fernández C. y Baptista L. (2010). Metodología de la Investigación. México: Mc Graw-Hill.

Kemmis, S. (2006). Mejorando la Educación Mediante la Investigación- Acción. Inicios y Desarrollo. Caracas, Venezuela: Laboratorio Educativo.

Lanz, R. (2006). Esa Incomoda Postmodernidad. México: Fondo Editorial Sentido.

Martínez, M. (1996). La Investigación Cualitativa Etnográfica en Educación. Manual Teórico - Práctico. México: Editorial Trillas.

Martínez, M. (2006). Ciencia y Arte en la Metodología Cualitativa. México: Editorial Trillas.

Morín, E. (1995). Introducción al Pensamiento Complejo. Barcelona: Gedisa.

Ochoa, H. (2018). Participación de los Padres de Familia como Corresponsables de la Educación de sus Hijos en una Institución Educativa del Callao. (Tesis de Maestría). Universidad San Ignacio de Loyola, Lima, Perú.

Perales, F., y Escobedo, M. (2016). La participación social en la educación: entre propuestas innovadoras y tradición educativa. Revista de Investigación Educativa, 18(1).

Pérez, G. (2002). Investigación cualitativa. Retos e interrogantes. II Técnicas y análisis de datos. Editorial La Muralla, S. A.

Rojas, B. (2007). Investigación Cualitativa. Fundamentos y Quehacer. Caracas: FEDUPEL

Sandín, E. (2003). Investigación Cualitativa en Educación. Barcelona. España: Mc Graw Hill.

Santizo, C. (2012). Gobernanza y cambio institucional de la Educación Pública Básica en México. México: UAM-Juan Pablos.

Teppa, S. (2012) Análisis de la Información Cualitativa y Construcción de Teorías. Barquisimeto - Venezuela: Editorial Horizonte, C.A.

Taylor, S. y Bogdan, R. (1994). Introducción a los métodos cualitativos de investigación. Buenos Aires, Argentina: Paidós.

Zurita, R. U. (2008). La participación social en la educación básica en México. Reflexiones en el marco de la Evaluación Nacional de la Participación Social en la Educación Básica, 2000-2006. Recuperado de http://www.redgestionescolar.org/file_biblio/participacionsocialOCE.pdf 